\title{
Schlußbericht über die Tätigkeit der deutschen Expedition zur Erforschung der Schlafkrankheit.")
}

\author{
Von \\ Prof. Dr. R. Koch.
}

Sese bei Entebbe (Uganda), den 25. April 1907.

Seit dem letzten Bericht haben wir uns hauptsächlich damit beschäftigt, den Einfluß, welchen das Atoxyl auf die Trypanosomen im Körper der Schlafkranken ausübt, nach den verschiedensten Richtungen hin zu studieren.

Bis dahin hatte sich ergeben, daß die Schwerkranken durch die Atoxylbehandlung zum großen Teil ganz erheblich gebessert wurden und daß die Trypanosomen in den Lymphdrüsen auf mindestens 30 Tage zum Verschwinden gebracht werden konnten.

Da manche von unseren Kranken sich bereits zwei bis drei Monate in Behandlung befanden, so entstand zunächst die Frage, ob nicht in gleicher Weise wie bei der Malaria dieser Zeitraum schon genügen würde, um die Blutparasiten vollständig zu vernichten. Zufällig traf es sich zur gleichen Zeit, daß unser Vorrat an Atoxyl nahezu erschöpft war und wir schon aus diesem Grunde die Behandlung nur in beschränktem Maße fortsetzen konnten. Es wurden also bei den meisten Kranken die Atoxylinjektionen auf mehr oder weniger lange Zeit ausgesetzt. Dabei ergab sich nun folgendes:

Die Besserung im Befinden der Kranken machte anfangs noch weitere Fortschritte, kam dann aber nach einigen Wochen zum Stillstand. Die vergrößerten Lymphdrüsen, bei denen, wie bereits früher berichtet wurde, eine deutliche Abnahme zu beobachten war, verkleinerten sich mehr und mehr, so daß 4 bis 5 Monate nach Beginn der Atoxylbehandlung nur noch wenige Kranke zu finden waren, deren Drüsen punktiert werden konnten. Und dieser Verkleinerung der Drüsen entsprechend verhielt sich auch die $A b-$ nahme der Trypanosomen in den Drüsen. Während der Atoxylinjektionen konnten mittels der Drüsenpunktion überhaupt keine Trypanosomen nachgewiesen werden. Aber nach dem Aussetzen der Behandlung erschienen sie in einigen Fällen wieder, und zwar am frühesten nach 11 Tagen. Die Zahl dieser Fälle nahm anfangs auch zu mit der Anzahl der Tage nach der letzten Atoxylinjektion, so daß gegen den 20. Tag nach der letzten Injektion bereits bei etwa $25 \%$ der Untersuchten Trypanosomen in den Drüssen wiedergefunden wurden. Es sah also so aus, als ob die Wirkung des Atoxyls nur eine vorübergehende sei. Dann trat aber ganz unerwartet ein Wechsel ein. Die Trypanosomen erschienen von da ab um so seltener, je längere Frist seit der letzten Injektion verstrichen war, und vom 60. Tage ab konnten wir nicht in einem einzigen Fall mehr Trypanosomen in den Drüsen auffinden. Bei den allermeisten Kranken waren, wie gesagt, die Drüsen zu dieser Zeit schon so verkleinert, daß sie für die Punktion nicht mehr geeignet waren,

1) Aus Deutsche Medizinische Wochenschrift, 1907, Nx. 46. 
aber es ließen sich doch noch 53 Fälle zusammenbringen, die punktiert werden konnten. Bei keinem einzigen konnten Trypanosomen nachgewiesen werden. Sehr merkwürdig war es, daß die Verkleinerung der Drüsen und das Verschwinden der Trypanosomen von der Zahl der Injektionen und von der Dauer der Behandlung unabhängig zu sein schienen. Sie traten auch bei solchen Kranken ein, welche nicht fortlaufend behandelt waren, sondern nur eine einzige Injektion von $0,5 \mathrm{~g}$ Atoxyl erhalten hatten. Derartige Fälle finden sich nicht wenige unter unseren Kranken, da viele derselben sich ganz unregelmäßig zur Behandlung einfinden und manche schon nach der ersten Injektion, die ihnen wegen der Schmerzhaftigkeit nicht zusagt, wegbleiben, um vielleicht erst nach Monaten wieder zum Vorschein zu kommen.

Schon in einem früheren Bericht hatte ich darauf hingewiesen daß manche Erscheinungen, welche bei der Atoxylbehandlung zur Beobachtung kommen, sich nur durch die Annahme einer Immunität infolge von Resorption der abgetöteten Trypanosomen erklären lassen. Auch die soeben erwähnte regelmäßige Abnahme der Drüsen und das vollständige Verschwinden der Trypanosomen, selbst nach einzelnen Injektionen, kann wohl nur in dieser Weise gedeutet werden. In bezug auf die Drüsen scheint diese Immunität auch für längere Zeit vorzuhalten. Es ist uns wenigstens kein Fall begegnet, in welchem nach länger als 60 Tagen Trypanosomen in den Drüsen wieder zum Vorschein gekommen wären.

Unter diesen Umständen war die Erwartung berechtigt, daß mit dem Verschwinden der vergrößerten Lymphdrüsen und der in ihnen enthaltenen Trypanosomen auch die Krankheit selbst abnehmen und schließlich verschwinden würde. Das war aber nicht der Fall. Wie bereits erwähnt wurde, kam die Besserung in dem Befinden der Kranken einige Zeit nach dem Aussetzen der Atoxylinjektion zum Stillstand, bei manchen Kranken trat sogar eine unverkennbare Verschlechterung ein. Daraus mußte der Schluß gezogen werden, daß die Krankheitsursache, $d$. h. die Trypanosomen, noch nicht vollständig beseitigt waren, sondern noch irgendwo im Körper stecken mußten. Aber wie sollten dieselben nachgewiesen werden, da die Drüsenpunktion nicht mehr ausführbar war und die Lumbalpunktion, an welche man in zweiter Linie denken mußte, bei unseren Kranken nicht angewendet werden konnte, weil sie sich derselben sofort widersetzt haben würden. Wir können hier nur solche Untersuchungsmethoden anwenden, welche die Eingeborenen. sich gutwillig gefallen lassen. Da blieb denn nichts weiter übrig, als die Blutuntersuchung, mit der wir früher recht ungünstige Erfahrungen gemacht hatten, wieder aufzunehmen. Dieselbe wurde so lange verbessert, bis sie zu befriedigenden Resultaten führte. Die Schwierigkeit der Blutuntersuchung beruht darauf, daß die Trypanosomen im Blute fast immer nur in sehr geringer Zahl vorhanden sind und außerdem nur anfallsweise auftreten, also nicht jederzeit anzutreffen sind. Unser früherer Mißerfolg hatte seinen Grund darin, daß wir in Ausstrichpräparaten zu geringe Mengen Blut untersucht hatten. Wenn man aber das Blut in möglichst dicker Schicht und unter Anwendung eines geeigneten Färbeverfahrens untersucht, dann lassen sich die Trypanosomen schon bei erstmaliger Untersuchung in einem großen Prozentsatz nachweisen. Werden dann die Untersuchungen in Zwischenräumen von einigen Tagen wiederholt, dann findet man schließlich in allen Fällen die Trypanosọmen. Allzu häufige Untersuchungen sind dazu glücklicherweise nicht nötig. Unter 75 Fällen einer Versuchsreihe lieferte schon die erste Untersuchung $40 \%$ positive Resultate, die zweite weitere $20 \%$. Bis zur fünften Untersuchung waren fast alle Fälle positiv, und nur in zwei Fällen waren sieben und selbst acht Untersuchungen zum Nachweis der Trypanosomen erforderlich. Das Verfahren ist, wenn eine größere Anzahl in Behandlung befindlicher Kranker kontrolliert werden muß, außerordentlich mühsam und zeitraubend; es gibt aber, wie gesagt, ganz zuver- 
lässige Resultate und ist das einzige, mit welchem wir den Verlauf der Krankheit und den Einfluß von Medikamenten bei unserem Krankenmaterial zu überwachen vermögen. Für schnelle Diagnosen bleibt natürlich die Drüsenpunktion immer noch das zweckmäßigste Verfahren.

Mit Hilfe der Blutuntersuchung wurde nun das Atoxyl in seinen verschiedenen Anwendungsweisen und auch andere in Frage kommende Medikamente auf ihre Wirksamkeit geprüft.

Während die Drüsenpunktionen keine wesentlichen Unterschiede in bezug auf die Anzahl der Atoxylinjektionen hatten erkennen lassen, vorausgesetzt, daß nur eine hinreichend große Dosis angewendet war, lehrte dagegen die Blutuntersuchung, da $\beta$ in dieser Beziehung doch deutliche Unterschiede bestehen.

Nach einer einmaligen Injektion von $0,5 \mathrm{~g}$ Atoxyl erschienen in einem Falle die Trypanosomen im Blute schon nach 5 Tagen. Nach den von uns in der Regel gegebenen Doppeldosen (je eine Dosis an zwei aufeinanderfolgenden Tagen) blieb das Blut nach dem Aussetzen des Mittels sehr viel längere Zeit frei von Trypanosomen, und zwar traten sie um so später auf, je länger und je regelmäßiger die Behandlung hatte durchgeführt werden können. In einigen Fällen erst nach 3 und selbst 4 Monaten. Bei einer geringen Zahl von Behandelten konnten bis jetzt überhaupt keine Trypanosomen trotz häufig wiederholter Untersuchungen gefunden werden.

Um nun aber zu besseren und namentlich dauernden Resultaten zu gelangen, wurde die Behandlung dahin abgeändert, daß mit der Dosis gestiegen wurde, und zwar gingen wir bis zu $1 \mathrm{~g}$ Atoxyl (gegenüber 0,5 g der früheren Behandlung), welches in Abständen von 7 bis 10 Tagen injiziert wurde. Nicht wenige Kranke entzogen sich sehr bald dieser stärkeren Behandlung, weil ihnen dieselbe zu schmerzhaft war und auch sonstige unangenehme Empfindungen verursachte, wie Übelkeit, Schwindelgefühl, kolikartige Schmerzen im Leibe. Da diese Beschwerden indessen nur vorübergehend waren, so wurde mit der starken Behandlung fortgefahren. Da stellte sich aber bei einigen Kranken ein Symptom ein, welches uns früher weder bei den unbehandelten Kranken noch bei denjenigen, welche nicht größere Dosen als $0,5 \mathrm{~g}$ erhalten hatten, jemals begegnet war. Es war dies eine Erblindung, welche sich in verhältnismäßig kurzer Zeit auf beiden Augen entwickelte. Anfangs hofften wir noch, daß dieses Symptom ebenso wie die anderen wieder vorübergehen würde, namentlich auch, da in Europa nach Atoxylbehandlung mehrfach vorübergehende Erblindung beobachtet ist. Leider trat aber bei unseren Kranken keine Besserung ein, und dieselben sind dauernd blind geblieben. Mit dem Augenspiegel ist an den erblindeten Augen keine Veränderung, auch nicht am Sehnerven; wahrzunehmen. Selbstverständlich haben wir, sobald wir die Überzeugung gewannen, daß die Erblindung durch die Atoxylbehandlung bedingt war, sofort mit der starken Behandlung aufgehört und sind wieder zu den früheren Halbgrammdosen übergegangen.

Es ist übrigens noch zu erwähnen, daß die Behandlung mit großen Atoxyldosen in keiner Weise bessere Resultate lieferte in bezug auf das Befinden der Kranken als die Behandlung mit mittelgroßen Dosen.

Einige Versuche, tägliche Injektionen von $1 / 2 \mathrm{~g}$ Atoxyl längere Zeit hindurch zu geben, scheiterten sehr bald daran, daß den Kranken diese Behandlung zu beschwerlich war und sie die Fortsetzung derselben verweigerten.

Da es ziemlich schwierig ist, bei den Eingeborenen eine Behandlung mit subkutanen Injektionen längere Zeit durchzuführen, so haben wir auch Versuche mit der inneren Anwendung des Mittels angestellt. Zuerst gaben wir das Atoxyl nur einigen Kranken innerlich und konnten uns bald davon überzeugen, daß Dosen von $1 / 2 \mathrm{~g}$ sehr gut vertragen 
werden und auch auf die Trypanosomen im Blute die erwünschte Wirkung ausüben. Als wir dann aber zu einem größeren Versuche mit 150 Kranken übergingen, stellte sich doch bald heraus, daß Halbgrammdosen nicht ausreichend sind; denn bei etwa 30\% der so Behandelten erschienen die Trypanosomen schon während der Behandlung wieder im Blute. Da größere Dosen, bis zu $1 \mathrm{~g}$, ebenso wie bei der Sublkutanbehandlung Vergiftungserscheinungen hervorriefen, so mußten wir auch diese Behandlungsmethode, welche die Bekämpfung der Schlafkrankheit sehr erleichtert haben würde, wieder fallen lassen.

Nachdem die erwähnten Verfahren sich nicht bewährt hatten, sind wir auf den ursprünglich eingeschlagenen Weg zurückgekommen und b e h a n de ln die $\mathrm{Kr}$ a $\mathrm{n}$ ken wieder mit Doppelinjektionen von $0,5 \mathrm{~g}$ Atoxylin zehn$t$ äg i g e $\mathrm{P}$ a u s e n. Auch die Leichtkranken, bei denen früher längere Pausen, bis zu 20 Tagen, gemacht wurden, erhalten jetzt ihre Doppelinjektionen zehntägig. Allerdings wird diese Behandlung viel länger durchgeführt werden müssen, als wir es bei unseren ersten Versuchen getan haben. Zurzeit haben wir schon wieder ziemlich viele Kranke, welche schon einige Monate in der angegebenen Weise behandelt wurden. Während der Behandlung sind noch bei keinem, obwohl schon Hunderte von Untersuchungen gemacht wurden, Trypanosomen gefunden. Von Zeit zu Zeit werden wir einige von diesen Kranken aus der Behandlung entlassen und dann mittels häufiger Blutuntersuchungen darauf prüfen, ob sie noch Trypanosomen haben.

Auch in bezug auf die von $\mathrm{E} \mathrm{h} \mathrm{r} \mathrm{l} \mathrm{i} \mathrm{c} \mathrm{h} \mathrm{in} \mathrm{letzter} \mathrm{Zeit} \mathrm{gemachte} \mathrm{wichtige} \mathrm{Entdeckung,}$ daß bei Tieren während der Atoxylbehandlung eine Gewöhnung der Trypanosomen an das Gift, die sogenannte Atoxylfestigkeit, eintreten kann, wird unsere jetzige Versuchsanordnung über kurz oder lang eine Auskunft geben müssen. Denn wenn auch beim Menschen und der von uns befolgten Behandlungsmethode die Trypanosomen gegen das Atoxyl unempfindlich werden, dann müssen sie allmählich schon während der Behandlung im Blute wieder zum Vorschein kommen, was aber, wie gesagt, bis jetzt noch in keinem Falle beobachtet ist. Auch bei unseren sonstigen Versuchen, die zum Teil, wie bei der inneren Behandlung, mit ungenügenden Dosen ausgeführt wurden, ist uns bis jetzt niemals eine Andeutung von einer sich entwickelnden Atoxylfestigkeit begegnet. Alle Kranken, bei denen sich während der Behandlung mit ungenügenden Dosen Trypanosomen im Blute wieder eingestellt hatten, verloren dieselben sofort und dauernd, wenn wir zur Subkutanbehandlung mit Halbgrammdosen und Doppelinjektionen übergingen.

Neben dem Atoxyl wurden von uns auch a n d e r e M i t t e 1 geprüft, welche sich bei Versuchen an Tieren den Trypanosomen gegenüber wirksam erwiesen hatten oder bei denen man wegen ihres Gehaltes an Arsenik eine solche Wirkung vermuten konnte.

So wurde vor allem die a $\mathrm{r}$ s e $\mathrm{n}$ i g e $\mathrm{S} \ddot{a} \mathrm{u} \mathrm{r}$ e in der Form des Natrium arsenicosum. sowohl subkutan als vom Magen aus versucht. Mehrere Kranke erhielten das Mittel in der Maximaldosis, zum Teil auch in noch größeren und wiederholten Dosen. Es wurde danach eine unverkennbare Wirkung auf die Trypanosomen beobachtet, doch blieb dieselbe hinter derjenigen des Atoxyls so weit zurück, daß die arsenige Säure als ein Ersatz für Atoxyl nicht in Frage kommen kann.

Zwei arsenhaltige Präparate, das $\mathrm{N} \mathrm{u}$ c l e o g e $\mathrm{n}$ von $\mathrm{H}$. R o s e n berg (Berlin) und das Arsenferratin von. G. F. Boehringer u. S. (Mannheim), zeigten entsprechend ihrem geringen Arsengehalt keinen merklichen Einfluß auf die Trypanosomen.

Von den Farbstoffen wurden bisher geprüft: Trypanxot (ein verbessertes Präparat, welches Geheimrat Ehrlich zur Verfügung gestellt hatte) und Afridolbla u 
(Dichlorbenzidir aus der Farbenfabrik von Fr. Bayer in Elberfeld). Die sublkutane Anwendung dieser Mittel ist recht schmerzhaft, und wir konnten sie deswegen nur in solcher Dosis geben, daß die Schmerzen eben noch erträglich waren. Eine öftere Wiederholung der Injektionen würde sich bei unserem Krankenmaterial nicht ausführen lassen. Bei diesen Mitteln wurde die Prüfung durch Drüsenpunktion vorgenommen. Während nun nach einer Injektion von $0,4 \mathrm{~g}$ Atoxyl nach wenigen Stunden die Trypanosomen aus den Drüsen verschwunden sind und lange Zeit wegbleiben, erschienen die Trypanosomen nach Injektion der Farbstofflösungen in keiner Weise beeinträchtigt. Wenn diese Mittel auch bei Tieren, denen man sie in erheblich größeren Dosen geben kann, allein oder in Kombination mit Atoxyl günstige Wirkungen haben können, so ist von ihrer Anwendung beim Menschen nach unseren Erfahrungen wohl kaum ein Nutzen zu erwarten.

Bei den außerordentlich zahlreichen Blutuntersuchungen, welche an unseren Kranken ausgeführt werden mußten, sind uns außer den Trypanosomen verschiedene and ere Blutpar a siten begegnet, welche ein gewisses Interesse beanspruchen.

Am häufigsten wurden Filarien, und zwar ausschließlich die Filaria perstans, gefunden. Dieser Parasit ist so häufig, daß es auf den Inseln und am nordwestlichen Ufer des Victoria-Njansa wohl kaum einen Eingeborenen gibt, der frei davon ist. Dies macht es erklärlich, daß frühere Forscher die Filaria als die Ursache der Schlafkrankheit angesehen haben. Irgendwelche Krankheitssymptome, welche man mit Sicherheit auf die Filarien hätte beziehen können, wurden auch bei den mit Filarien stark behafteten Eingeborenen nicht beobachtet. Elephantiasis, welche man anderwärts mit Filarien in ursächlichen Zusammenhang gebracht hat, kommt hier nicht vor. Bekanntlich nimmt man an, daß die Filarien durch die Stiche der Moskitos übertragen werden. Da nun hier fast jeder Mensch Filarien hat, so muß die Ansteckungsgelegenheit eine sehr häufige und kaum vermeidbare sein, und es war deswegen zu befürchten, daß auch die Mitglieder der Expedition und die von uns mitgebrachten Küstenleute sehr bald mit Filarien behaftet sein würden, denn es ist unmöglich, hier die Stiche der Moskitos vollkommen $\mathrm{zu}$ vermeiden. Bis jetzt ist glücklicherweise aber eine derartige Infektion nicht vorgekommen. Auch bei einem Missionar, welcher sich schon seit Jahren in Uganda aufhält und dessen Blut wegen Malaria untersucht wurde, fanden sich keine Filarien. Merkwürdigerweise fehlen dieselben auch bei einigen eingeborenen Oberhäuptlingen. Da alle diese Personen schon oft von Moskitos gestochen sind, so liegt die Vermutung nahe, daß in hiesiger Gegend und für die Filaria perstans eine andere Art der Übertragung statthat als diejenige durch Moskitos.

Malariaparasiten sind hier auch recht häufig. Die Zahl der Fälle, bei denen Malariaparasiten im Blute nachgewiesen wurden, schwankt, je nach der Gegend, aus welcher die Leute stammen, zwischen 20 und 50\%. Zum allergrößten Teil gehören die Parasiten der tropischen Malaria an. Quartanaparasiten sind nicht selten, Tertianaparasiten wurden dagegen nur in wenigen Fällen angetroffen. Merkwürdigerweise werden die Malariaparasiten durch die Atoxylbehandlung bei weitem nicht so beeinflußt wie die Trypanosomen. Sie scheinen an Zahl abzunehmen, da man bei den Behandelten meistens nur einzelne Parasiten und Gameten trifft, aber sie werden doch nicht ganz zum Verschwinden gebracht. Umgekehrt werden die Trypanosomen durch das Chinin nicht merklich beeinflußt. Beide Mittel verhalten sich daher den betreffenden Parasiten gegenüber spezifisch,

Weniger häufig als die beiden soeben erwähnten Parasiten wurden R e c u r r e n s s p i r o c h a e t e n gefunden, nämlich bei 14 Eingeborenen, und zwar bei elf Erwachsenen 
und drei Kindern. Die Erwachsenen hatten nur vereinzelte Spirochaeten im Blute und zeigten keine auffallenden Krankheitssymptome; nur bei einigen war die Körpertemperatur für kurze Zeit erhöht. Ganz anders gestaltete sich aber die Krankheit bei den Kindern. Diese waren mehrere Tage schwerkrank, in regelmäßigen Zwischenräumen wiederholten sich diese Anfälle, und, was besonders bemerkenswert war, sie hatten in ihrem Blute sehr zahlreiche Spirillen, ganz so, wie es beim europäischen Recurrensfieber der Fall ist.

Das Vorkommen von Recurrens gab Veranlassung, einige Nachforschungen in bezug auf Ornithodoruszecken, welche bekanntlich die Überträger der Rekurrensspirochaeten sind, anzustellen. Sie wurden denn auch in verschiedenen Hütten nachgewiesen, namentlich auch in dem Wohngebäude der Mission. Aus letzterem erhielten wir sechs Zecken, von denen zwei mit Spirochaeten infiziert waren. Ursprünglich war dies Gebäude für uns als Wohnung in Aussicht genommen. Es war mir aber von vornherein verdächtig vorgekommen, weil einige Räume desselben von Eingeborenen benutzt werden. Ich hatte es deswegen vorgezogen, in dem Missionsgebäude nur unsere Vorräte unterzubringen.

Auf der Herreise konnten wir die beiden Grenzstationen des deutschen Schutzgebiets am Victoria-Njansa, Shirati und Buksoba, besuchen und dort Erkundigungen über das Vorkommen der Schlafkrankheit einziehen. Dieselben ergaben, daß damals auf beiden Stationen von Schlafkrankheit noch nichts bekannt war, und ich konnte dementsprechend berichten, daß bis dahin das deutsche Gebiet am Victoria-Njansa noch seuchenfrei sei.

So verhält es sich augenblicklich aber nicht mehr.

Vor einigen Wochen erhielt ich die Nachricht, daß in der Landschaft Mageta, $40 \mathrm{~km}$ südöstlich von Shirati an den Quellen des Shririaflusses, mehrere verdächtige Kranke angetroffen worden seien. Drei Kranke wurden nach Shirati geschafft und Blutproben derselben zur Untersuchung eingesandt. In dem Blute von zweien dieser Kranken wurden Trypanosomen nachgewiesen. Damit ist das Vorkom men der Seuche in dem Shiratibezirkfestgestellt. Wenn der Herd der Krankheit sich im Quellgebiet des Flusses, also entfernt vom Ufer des Sees befindet, dann würde dies ein ganz außergewöhnliches Vorkommnis sein. Sollten weitere Anfragen, welche ich nach Shirati gerichtet habe, diese Vermutung bestätigen, dann wäre eine Untersuchung an Ort und Stelle notwendig.

$\mathrm{A} u \mathrm{ch}$ i $\mathrm{m} \mathrm{B} \mathrm{k}$ ob a bezirk, und zwar in der Landschaft Kisiba, welche der englischen Grenze benachbart ist, hat die $S$ e u che bereitsfesten Fu $\mathrm{Bg}$ $\mathrm{f}$ a $\beta$ t. Aus jener Landschaft sind in den letzten Monaten nach und nach 58 Eingeborene hierhergekommen, um sich wegen Schlafkrankheit behandeln zu lassen. Unter diesen Leuten befinden sich 28, bei denen Trypanosomen nachgewiesen werden konnten. Die übrigen sind als Ruderer und zur Pflege der Kranken mitgefahren. Von den 28 Kranken sind 24. Männer und 4 Frauen. Die Bewohner von Kisiba sind eifrige Seefahrer und unternehmen mit ihren Booten häufige Reisen nach Uganda und nach den Seseinseln. Daher kommt es, daß die kranken Männ'er schon sämtlich Uganda oder die Seseinseln früher besucht haben und sich dort auch infiziert haben können. Aber die vier Frauen haben Kisiba niemals verlassen und können nur dort infiziert sein. Die Leute kennen auch die Glossina palpalis, von welcher ihnen lebende Exemplare gezeigt wurden, sehr gut, und sie sagen, daß diese Fliegen bei ihnen am Seeufer vorkommen. In den verschiedenen Dörfern sollen sich noch viele Schlafkranke befinden. Es kann somit kein Zweifel 
bestehen, daß a uch in Kisiba ein Seucheherd, und anscheinend ein ziemlich bedeutender, sich gebildet hat. Auch hier wird eine Untersuchung des Herdes erforderlich sein, welche ich, sobald die Regenzeit beendet ist, zu unternehmen gedenke.

Schließlich habe ich noch über den Abschluß eines Versuches zu berichten, welcher in einem früheren Berichte (Muanza, den 31. Juli 1906) erwähnt ist. Es handelt sich um die Insel Sijawanda bei Muanza, auf welcher zahlreiche Glossinen vorkommen. Diese Insel sollte teilweise durch Abholzen fliegenfrei gemacht werden. Das Abholzen ging etwas langsam vonstatten, weil nur wenige Arbeiter zur Verfügung standen. Bei der Abreise der Expedition von Muanza war deswegen das Experiment noch nicht beendigt, und ich habe daher Herrn Oberarzt Dr. R a d l of $f$ in Muanza gebeten, den weiteren Verlauf zu überwachen, was derselbe auch in dankenswerter Weise getan hat. Er teilte mir am 14. Dezember 1906 mit, daß die Insel bis auf einen kleinen Taleinschnitt, welcher seine Vegetation behielt, abgeholzt wurde. An einer Stelle des Ufers blieben auch die im Wasser wachsenden Ambatschbüsche stehen. Nachdem dies geschehen, wurde die Insel von Herrn Dr. $\mathrm{R}$ a d l of f wiederholt besucht, und es wurde festgestellt, daß niemals in dem ganzen abgeholzten Gebiet Glossinen angetroffen wurden. Dagegen fanden sich an den Ambatschbüschen einige wenige und zahlreiche Glossinen in dem nicht abgeholzten Einschnitt. Der Versuch ist somit vollständig gelungen und liefert wiederum ein Beispiel dafür, daß die Glossinen, wo es darauf ankommt, leicht zu vertreiben sind. Das Abholzen ist auch keine kostspielige Maßregel. Es wird am See von den Dampfschiffen sehr viel Brennholz gebraucht, und bei unserem Versuch hätten die Arbeitskosten durch etwaige Verwertung des gewonnenen Holzes als Brennholz nach einer angestellten Berechnung reichlich gedeckt werden können.

Sese, den 5. September 1907.

Aus Kisiba habe ich die Nachricht erhalten, daß bis Ende August 365 Kranke in Behandlung genommen waren. Dieselben waren fast sämtlich aus dem Sultanat Kisiba, während aus dem benachbarten Sultanat Bugabu, wo wir bei unserem Durchmarsch auch zahlreiche Fälle von Schlafkrankheit angetroffen hatten, sich nur wenige Kranke eingefunden haben. Sobald Stabsarzt $\mathrm{K} \mathrm{u} \mathrm{d} \mathrm{i} \mathrm{c} \mathrm{k} \mathrm{e} \mathrm{einen} \mathrm{Lazarettgehilfen} \mathrm{er-}$ halten hat, was bis jetzt noch nicht möglich zu machen war, beabsichtigt er selbst nach Bagabu zu gehen, die Kranken aufzusuchen und in das Lager bei Kigarama zu bringen. Sollte sich aber herausstellen, daß die Bugabuleute ihr Land nur sehr ungern verlassen, dann wird es geraten sein, auch in Bugabu ein Krankenlager zu errichten und mit einem Arzt zu besetzen.

Alle Bemühungen, in demjenigen Teil von Kisiba, wo die Krankheit vorzugsweise herrscht, die Glossina palpalis aufzufinden, sind vergeblich gewesen, und es kann wohl jetzt schon als sicher angenommen werden, daß die Fliege in diesen Gegenden nicht vorkommt. Dementsprechend konnte auch immer wieder festgestellt werden; daß die Kranken sich nicht in Kisiba, sondern in Uganda, wo sie sich längere Zeit aufgehalten hatten, infiziert haben. Die einzige Ausnahme machten einige Frauen, deren Zahl jetzt 15 beträgt. Diese hatten Kisiba niemals verlassen und können nur hier den Krankheitskeim aufgenommen haben. Nun ist aber weiter festgestellt, daß die Frauen sämtlich verheiratet sind, daß ihre Männer entweder an Schlafkrankheit gestorben sind oder, sofern sie noch leben, an dieser Krankheit leiden. Besonders wichtig für die Deutung dieses Vorkommens von Schlafkrankheit bei verheirateten Frauen ist, daß 
in einem Falle ein Mann mit Trypanosomiasis drei Frauen hat und daß alle drei Franen nachgewiesenermaßen ebenfalls an Trypanosomiasis leiden; eine davon ist bereits schwerkrank. Hierausmußgeschlossen werden, d a Bdie Infektion nur durch den ehelichen Verkehr in diesem Fallebewirkt sein $\mathrm{k}$ a $\mathrm{n}$. Dasselbe gilt dann auch von den übrigen Frauen; denn wenn irgendeine andere Ursache, z. B. blutsaugende Insekten, die Krankheit in Kisiba von Infizierten auf Gesunde übertragen würde, dann müßten nicht ausschließlich Frauen erkranken, deren Männer an Trypanosomiasis leiden, sondern auch Frauen von gesunden Männern, ferner unverheiratete Frauen, Kinder und ältere Leute, welche doch auch in enger Berührung mit den Infizierten leben.

Überhaupt sind die Verhältnisse in Kisiba, wo es viele Kranke, aber keine Glossinen gibt, außerordentlich lehrreich in bezug auf die Frage, ob die Schlafkrankheit auch auf andere Weise als durch die Glossina palpalis übertragen werden kann. Kisiba ist sehr reich an blutsaugenden Insekten. Moskitos verschiedener Art, Stomoxys, Tabanus sind vertreten, außerdem Zecken, welche den Menschen angreifen, auch Ornithodorus. Und doch ist nicht ein einziger Fall bekannt geworden, der durch derartige Krankheitsüberträger infiziert wäre. Die Infektion durch den geschlechtlichen Verkehr betreffend, sei noch darauf hingewiesen, daß bei einer anderen Trypanosomenkrankheit, der Dourine oder Beschälkrankheit, dies der einzige Weg ist, auf welchem die Infektion zustande kommt.

Auf einer Exkursion nach der Halbinsel Buninga traf ich zufällig ein Lager von Gummisammlern, das aus 18 Eingeborenen mit einem Aufseher bestand. Darunter befanden sich 15 Männer aus Deutsch-Kisiba. Von diesen Leuten erfuhr ich, daß allein auf Buninga sieben derartige Lager bestehen mit 80 bis 100 Männern aus Kisiba. Da der Gummi aus dem Safte der Gummiliane (Landolphia) gewonnen wird, die in den Urwäldern am Ufer des Sees wächst, wo gleichzeitig die Glossina palpalis massenhaft vorkommt, so sind die Gummisammler der Infektion ganz besonders ausgesetzt. Sie gehörten denn auch zu den ersten Opfern der Seuche, und nachdem sie weggestorben waren, hat die Gummigewinnung eine Zeitlang ganz aufgehört, da sich niemand mehr zu diesem gefährlichen Gewerbe trotz guter Bezahlung hergeben wollte. Jetzt scheint man aber wieder Leute, und besonders in Deutsch-Kisiba, gefunden zu haben, welche sich in Unkenntnis der Gefahr anwerben lassen und ebenso wie die früheren Sammler der Seuche zum Opfer fallen werden. Als ich die Sammler untersuchte, fand ich mehrere, welche die Symptome der Infektion bereits in unverkennbarer Weise zeigten. In kurzer Zeit werden sie zu schwach sein, um noch arbeiten zu können. Sie gehen dann in die Heimat zurück, und andere, durch den hohen Verdienst angelockt, werden an ihre Stelle treten. Man erfährt aus diesem Beispiel, wie es kommt, daß sich in Kisiba so viele Schlafkranke befinden.

In Shirati sind zurzeit Stabsarzt $\mathrm{F}$ e l $\mathrm{d}$ m a $\mathrm{n} \mathrm{n}$ und Oberarzt B r e u e r mit der Bekämpfung der Schlafkrankheit beschäftigt. Ersterer hat mir vor kurzem berichtet, daß bis zum 6. August 143 Schlafkranke sich auf der Station Shirati eingefunden haben und daß, wenn sich die Krankmeldungen in gleicher Weise fortsetzen, die Zahl 200 bald erreicht sein wird. Er hat sich deswegen veranlaßt gesehen, ein Krankenlager, ähnlich dem von Kisiba, zu errichten, dessen Leitung vorläufig Oberarzt B r e u e r übernehmen wird. Stabsarzt $\mathrm{F}$ e l d $\mathrm{m}$ a $\mathrm{n}$ n wird später den südlichen Teil des Bezirks Shirati, insbesondere die Umgebung der Mori- und der Marabucht, bereisen, um die Ausbreitung der Seuche auch in diesen Gegenden festzustellen. Da die Zahl der Kranken jetzt schon erheblich höher ist, als ich in meinem vorigen Bericht angenommen habe, und auch noch weiter wachsen wird, wenn der ganze Bezirk demnächst nach Kranken 
abgesucht wird, so ist es notwendig, daß in Shirati ein Arzt und ein Lazarettgehilfe dauernd stationiert werden.

Die Untersuchungen über Substanzen, welche sich im Tierversuch wirksam gegen die Trypanosomen erwiesen hatten und aus diesem Grunde als Ersatz des Atoxyls oder zur Unterstützung desselben in Frage kommen konnten, wurden fortgesetzt.

Außer dem ,Afridolblau“ (Dichlorbenzidin + H-Säure) von der Farbenfabrik Fr. Bayer u. Co., Elberfeld, über welches ich früher berichtet habe, wurde noch ,A f r id o I vi o l e t t" (Diamidodiphenylharnstoff $+\mathrm{H}$-Säure), ein Präparat derselben Fabrik, geprüft. Dasselbe war aber auch nicht imstande, in den höchsten noch anwendbaren Dosen einen merkbaren Einfluß auf die Trypanosomen auszuüben.

Es wurden dann weiter zwei Präparate untersucht, welche von der Fabrik von Leopold Casella \& Co. in Frankfurt a. M. auf Veranlassung von Geheimrat E h r li c h eingesandt waren. Es sind dies das öls a u re Pararosanilin und das Parafuchsina z et a.t. Beide Mittel wurden innerlich gegeben, da nach früheren Erfahrungen eine subkutane Anwendung sich längere Zeit hindurch nicht hätte ausführen lassen. In täglichen Dosen von $1 / 2 \mathrm{~g}$ wurde das ölsaure Pararosanilin gut vertragen, auch ein volles Gramm konnte noch gegeben werden. Das Fuchsinpräparat machte dagegen schon in Halbgrammdosen einigen Kranken solche Beschwerden (Magenschmerzen und Erbrechen), daß es ausgesetzt werden mußte, und Grammdosen ertrugen nur einige Kranke. Die Präparate wurden, soweit es sich ausführen ließ, zwei Wochen lang täglich gegeben und zugleich das Blut auf Trypanosomen untersucht. Dabei stellte sich hèaus, daß Trypanosomen im Blute auch während der Behandlung öfters nachgewiesen werden konnten, was beim Atoxyl niemals vorkommt. Zum Schluß wurde dann noch die Drüsenpunktion vorgenommen und auch dabei die Trypanosomen gefunden. Eine deutliche Wirkung auf die Trypanosomen konnte somit nicht nachgewiesen werden. Wenn diese Präparate im Tierversuch eine ausgesprochene Wirkung gezeigt haben, so liegt dies daran, daß man Tieren bedeutend größere Dosen geben kànn als dem Menschen. Zum Ersatz des Atoxyls sind sie bei der Behandlung der Schlafkranken nicht geeignet.

In bezug auf die in meinem früheren Berichte erwähnten Erblindungen, welche im Laufe der Atoxylbehandlung eintreten können, bemerke ich, daß dieselben nicht mehr vorgekommen sind, seitdem wir auf Halbgrammdosen zurückgegangen sind. Daraus geht wohl deutlich hervor, daß diese Unglücksfälle in der Tat durch die Atoxylbehandlung bedingt sind. Insgesamt haben wir 22 Fälle von Erblindung beobachtet, welche wegen ihrer Wichtigkeit in dem ausführlichen Bericht, namentlich in bezug auf die Dauer der Behandlung und Dosierung des Mittels, beschrieben werden sollen.

Durch unsere früheren Untersuchungen war festgestellt, daß eine zwei Monate lang durchgeführte Atoxylbehandlung zwar eine kräftige Wirkung hat, indem die klinischen Symptome ganz erheblich gebessert und die Trypanosomen zum Verschwinden gebracht wurden, daß aber diese Zeitdauer doch noch nicht ausreichend war, weil bei der Mehrzahl der Fälle die Trypanosomen, wenn auch erst nach Monaten und in langen Zwischenräumen, wieder zum Vorschein kamen. Es war daher notwendig, zu untersuchen, ob Atoxylkuren von längerer Zeitdauer bessere Erfolge haben würden. Zu diesem Zwecke wurde versucht, die Kranken eine längere Zeit hindurch regelmäßig zu behandeln. In ganz exakter Weise hat sich dies aber in keinem Falle durchführen lassen, weil die Eingeborenen, obwohl sie für medikamentöse Behandlung jeder Art sehr eingenommen sind, doch keine Ausdauer besitzen. Sobald sie sich einigermaßen gebessert fühlen, 
oder wenn sich die Kur in die Länge zieht und ihnen langweilig wird, damn brechen sie dieselbe $a b$ und laufen davon. Sehr viele von unseren Kranken, welche von auswärts gekommen waren, mußten auch deswegen vorzeitig aufhören, weil sie ihr Besitztum nicht länger unbeaufsichtigt lassen konnten, oder weil ihnen die Mittel zum Unterhalt ausgingen. Dabei mußte natürlich mit der Möglichkeit gerechnet werden, daß die Leute wieder zu derselben Stelle zurückkehrten, wo sie früher erkrankt waren, und daß sie daselbst von neuem infiziert werden konnten. Hiergegen ließ sich nichts tun, da wir irgendwelchen Zwang auf die Leute selbstverständlich nicht ausüben wollten und auch unter den hiesigen Verhältnissen gar nicht hätten ausüben können. Ganz anders werden sich aber die Verhältnisse gestalten, wenn es gilt, die Schlafkrankheit regelrecht zu. bekämpfen; dann wird man, auch schon im eigenen Interesse der Kranken, auf eine genaue Durchführung der Atoxylbehandlung bestehen müssen.

Wenn somit unser Beobachtungsmaterial auch in bezug auf die einzelnen Fälle zu wünschen übrig läßt, so wird doch dieser Mangel durch die große Zahl der uns zur Verfügung stehenden Beobachtungen einigermaßen wieder ausgeglichen, und es $l$ a s s e $\mathbf{n}$ sichausdiesemumfangreichen Materialfolgende Tatsachen a bleiten.

Bei einem Schlafkranken, welcher nach der von uns befolgten Methode mit Atoxyl behandelt wird, treten niemals während der Behandlung, und wenn dieselbe bis zu 10 Monaten dauert, Trypanosomen im Blute auf. Weitere Untersuchungen müssen lehren, um wieviel länger dieser Zeitraum zu bemessen ist. Auf jeden Fall hat sich während der angegebenen Zeit kein Anzeichen von einer Gewöhnung der Trypanosomen an das Atoxyl bemerkbar gemacht, und es hat sich die sogenannte Atoxylfestigkeit, welche beim Tierversuch beobachtet wurde, bei der von uns befolgten Behandlungsmethode am Menschen nicht eingestellt. Man is t s o mit i m s t a n d e, Mens chen, we lche an Trypanosomiasis leiden, mindestens lo Monate lang in bezug a uf ihr Blutfreivon Trypanosomenzuhalten und damit zu bewirken, da $\beta$ siefür die Infektion vonGlossinen und infolgedessen für die Ausbreitung der Krankheit ungeeignet, d. h. ungefährlich werden. Diese Eigenschaft des Atoxyls allein würde schon ausreichend sein, um es im Kampfe gegen die Schlafkrankheit mit Erfolg verwenden zu können. Aber es leistet noch erheblich mehr, denn nach dem Aussetzen der Atoxylbehandlung treten zwar bei einer gewissen Anzahl von Kranken nach längerer oder kürzerer Zeit wieder Trypanosomen im Blute auf. Aber je länger und je regelmäßiger die Behandlung durchgeführt und in je früherem Stadium der Krankheit sie begonnen wurde, um so geringer wird die Zahl derjenigen, welche durch die Atoxylbehandlung nicht vollständig von den Trypanosomen befreit werden. Bei den Leicht$\mathrm{kr}$ a $\mathrm{nk}$ e $\mathrm{n}$ werden daher die besten Erfolge erzielt, und man kann wohl behaupten, $\mathrm{da} B$ die weit überwiegende Mehrzahl derselben durch eine Kur von 4 bis 6 Monaten dauernd von Trypanosomen befreit, also, da sie außerdem nicht die geringsten Krankheitssymptome aufweist, als vollkommen geheilt anzusehen ist. Weniger günstig gestalten sich die Erfolge bei den $\mathrm{Schw}$ e $\mathrm{kr}$ a $\mathrm{nk}$ en. Auch unter diesen finden sich nicht wenige, welche durch die Atoxylbehandlung anscheinend hergestellt werden und während der Zeit, in welcher wir sie beobachten konnten, auch unverändert geblieben sind. Andere aber haben sich nach dem Aussetzen des Atoxyls wieder verschlechtert, und manche, welche sich bereits für geheilt hielten und sich deswegen der Behandlung zu früh entzogen haben, sind zugrunde gegangen. Genauere Angaben hierüber, sowie die Mitteilung der betreffenden Beobachtungsreihen und Zahlen muß ich mir für den ausführlichen Bericht vorbehalten. Nur über die Mortalität der unbehandelten und der 
mit Atoxyl behandelten Schlafkranken möchte ich hier einige Ziffern kurz anführen, weil dieselben am besten geeignet sind, den günstigen Einfluß der Atoxylbehandlung zu zeigen.

Auf der Missionsstation Bumangi sind bei einem durchschnittlichen Krankenbestand von 20 Schlafkranken im Laufe von 4 Jahren 212 Todesfälle vorgekommen, das gibt für $100 \mathrm{Kranke}$ und auf $1 \mathrm{Jahr}$ berechnet 265. Auf der Missionsstation Kisubi sind bei einem durchschnittlichen Bestand von 80 Kranken in 3 Jahren 687 gestorben. Auf 100 Kranke kommen also jährlich 287 Todesfälle. Die Mortalität auf beiden Stationen war nahezu gleich. Sie ist auch nicht etwa anfangs niedriger gewesen und erst infolge der Anhäufung der Kranken gestiegen, denn in Bumangi starben schon im ersten Jahre 52, was fast genau dem jährlichen Durchschnitt entspricht. Diese hohen Mortalitätsziffern bedeuten, daß die Schlafkranken in dem Stadium, in welchem sie ins Lazarett gelangen, nur noch 4 bis 5 Monate durchschnittlich zu leben haben. Und in der Tat sind auf beiden Stationen früher, d. h. vor der Atoxylbehandlung, sämtliche Schlafkranke, mit ganz wenigen Ausnahmen, bei denen wohl die Diagnose nicht gestimmt hat, gestorben.

Die Zahl unserer Kranken beläuft sich auf 1633. Davon sind im Laufe von 10 Monaten gestorben 131, also $8 \%$. Unser Krankenmaterial weicht aber insofern von demjenigen der Missionsstationen ab, als sich unter dem unsrigen eine größere Zahl von Leichtkranken befindet. Wenn wir aber auch ausschließlich unsere Schwerkranken in Betracht ziehen, dann ergibt sich auf 374 eine Mortalität von 78 oder 22,9\%. Darunter befinden sich jedoch auch die ganz ungenügend Behandelten, welche nur eine oder wenige Atoxylinjektionen erhalten haben. Wollte man diese noch abrechnen, dann würde die Mortalität kaum halb so hoch sein. Die Mortalität bei unseren mit Atoxyl behandelten Schwerkranken beträgt also nicht ganz den 10., vielleicht nur den 20. Teil von derjenigen der nicht mit Atoxyl behandelten Schlafkranken. Daraus geht doch aber mit aller Bestimmtheit hervor, daß durch eine geeignete Atoxylbehandlung sehr vielen Schlafkranken das Leben gerettet werden kann.

Es ist wohl möglich, daß im Laufe der Zeit andere Mittel gefunden werden, welche noch mehr Erfolg haben als das Atoxyl und dann an dessen Stelle treten können. Aber das Atoxyl ist, wenn auch kein unfehlbares Mittel, so doch eine so gewaltige Waffe im Kampfe gegen die Schlafkrankheit, daß man es jetzt schon so viel als irgend möglich dafür ausnutzen muß.

Unter Zuhilfenahme unserer bisherigen Erfahrungen wird sich die Bekämpfung der Schlafkrankheit folgenderma $\beta$ en gestalten.

Es sind zunächst stehende Lager zu errichten, in welchen die Kranken untergebracht werden. Die Anzahl derselben richtet sich danach, wieviel Kranke aufzunehmen sind, und weiter nach den Entfernungen, welche beim Aufsuchen und beim Transport der Kranken in Betracht kommen.

Das Lager muß, damit die Verpflegung der Kranken nicht auf Schwierigkeiten stößt, in niçht zu großer Entfernung von bewohnten Orten, und insbesondere an einem Platze errichtet werden, wo es keine Glossinen gibt. Es steht unter der Leitung eines Arztes, dem ausreichende europäische Hilfskräfte zur Verfügung zu stellen sind. Es ist nicht darauf zu rechnen, daß die Kranken sämtlich freiwillig kommen. Sie müssen aufgesucht werden, und es ist dabei besonders wichtig, die in den ersten Stadien befindlichen Kranken aufzufinden, welche sich noch nicht krank fühlen, überall hingehen und so vorzugsweise geeignet sind, die Krankheit zu verschleppen. In dieser Beziehung genügt es nicht, die Verdächtigen auf vergrößerte Lymphdrüsen zu untersuchen, es muß auch die Blutuntersuchung nach der von uns befolgten und sehr bewährten Methode gemacht werden. Wie wichtig gerade dieser Punkt ist, lehrt folgendes Beispiel. 
52 junge, kräftige Männer, welche als Ruderer die Fahrt von Entebbe nach Sese gemacht und dabei fast ununterbrochen $12 \frac{1}{2}$ Stunden gerudert hatten, wurden untersucht. Sie selbst hielten sich für gesund, und jeder hätte sie in Anbetracht ihrer körperlichen Leistungen auch für gesund gehalten. Bei 11 wurden mehr oder weniger vergrößerte Lymphdrüsen am Halse gefunden. Bei einer einmaligen Blutuntersuchung stellte sich heraus, daß sieben Trypanosomen im Blute hatten, und zwar fünf mit vergrößerten Lymphdrüsen und zwei mit normalen Drüsen. Nach unseren Erfahrungen werden bei einer einmaligen Blutuntersuchung etwa 50\% der Trypanosomenträger gefunden, und es ist anzunehmen, daß bei wiederholten Untersuchungen auch in diesem Falle die doppelte Zahl gefunden sein würde, mithin zehn Leute mit Trypanosomen im Blute bei vergrößerten Lymphdrüsen und vier Trypanosomenträger mit normalen Lymphdrüsen. Nebenbei bemerkt, hatten von diesen 52 anscheinend gesunden Leuten 47 Filaria perstans, 26 Malariaparasiten und 2 Recurrensspirillen in ihrem Blute. So sieht die noch für gesund geltende hiesige Bevölkerung aus.

Dies ist übrigens nicht das einzige Beispiel für das Vorkommen von Trypanosomenträgern mit normalen Lymphdrüsen, über welches ich verfüge. Derartige Leute kommen gar nicht selten vor, und es würde deswegen verkehrt sein, die Bekämpfung der Schlafkrankheit, wie vorgeschlagen ist, ausschließlich auf den Befund der vergrößerten Lymphdrüsen zu begründen.

Sämtliche Kranke, welche in dem Lager angesammelt werden, sind einer mindestens 4 Monate währenden regelmäßigen Atoxylbehandlung zu unterwerfen. Sollte ein wirksameres Mittel als das Atoxyl gefunden werden, so tritt dieses an seine Stelle. In der hier skizzierten Bekämpfung der Schlafkrankheit wird dadurch nichts geändert. Nach Beendigung der Kur muß durch wiederholte Blutuntersuchungen das dauernde Verschwinden der Trypanosomen festgestellt werden. Die Kranken müssen so lange im Lager bleiben, bis anzunehmen ist, daß an ihrem Wohnorte nach Entfernung aller Trypanosomenträger die Glossinen frei von Infektionsstoff geworden sind. Hierüber wissen wir augenblicklich leider noch nichts; aber es wird nicht schwierig sein, im Laufe der Zeit Beobachtungen zu sammeln, aus denen man den fraglichen Zeitpunkt bestimmen kann. Vorläufig würde ich mindestens $1 \mathrm{Jahr}$, womöglich $2 \mathrm{Jahre}$ dafür erforderlich halten.

Die Einrichtung von Lagern gilt für alle Stellen, wo Schlafkrankheit vorkommt. Aber außerdem kommen noch weitere Maßnahmen in Betracht, welche je nach den örtlichen Verhältnissen verschieden sind.

So sind gegen die Einschleppung aus anderen Gegenden Verkehrsbeschränkungen, Grenzsperren, internationale Vereinbarungen erforderlich. In Kisiba, wo es fast nur aus Uganda eingeschleppte Fälle gibt, wird dies sogar die wichtigste Maßregel sein. Zum Teil gilt dies aber auch für den Shiratibezirk in bezug auf die Einschleppung vom benachbarten englischen Gebiet und am Tanganjika wegen der Einschleppung vom Kongostaat her, welche nach Feld manns Bericht dort eine wichtige Rolle spielt.

In Gegenden, wo eine an Zahl geringe Bevölkerung in ausgedehnten, mit Glossinen besetzten Gebieten lebt, wird neben der Atoxylbehandlung der Erkrankten die Versetzung dieser Bevölkerung in glossinenfreie Gegenden das einfachste Mittel zu ihrer Rettung sein. Dieses Verfahren wird voraussichtlich für den dünn bevölkerten Küstenstrich, nördlich von Shirati, das zweckmäßigste sein.

In anderen Gegenden wird es sich empfehlen, durch A b h o I z e n d e r St e Il e n, wo di e Glos sin a le bt, diese zu vertreiben. Doch wird diese Maßregel wohl immer nur eine räumlich beschränkte Anwendung finden können. Sie soll beispielsweise, wie in Shirati verabredet wurde, am Seeufer, in der Umgebung der Station, wo vereinzelte Glossinen gefunden wurden, ausgeführt werden. 
Gegen die Glossinen läßt. sich dadurch etwas ausrichten, daß man ihnen ihre regelmäßige Nahrungszufuhr abschneidet. Diese Insekten müssen alle 2 bis 3 Tage Gelegenheit haben, sich mit dem Blut von Wirbeltieren zu füllen. Woher sie dieses Blut nehmen, kann man durch Untersuchung ihres Mageninhaltes leicht ermitteln. Auf diese Weise haben wir festgestellt, daß an den Ufern des Victoria-Njansa die Glossinen fast nur von Krokodilblut leben. Man würde ihnen also ihre Existenzmöglichkeit sehr einschränken, wenn die Krokodile ausgerottet oder doch ihre Zahl erheblich gemindert würde, und das ist nicht schwierig, wenn man darauf ausgeht, den Nachwuchs zu verhindern. Die Krokodile haben ganz bestimmte Brutplätze, welche sie immer wieder aufsuchen. Den Eingeborenen sind diese Plätze bekannt, und sie können durch Prämien veranlaßt werden, die Krokodileier zu sammeln und abzuliefern. In früheren Zeiten ist dies, wie ich erfahren habe, auch an dem zum deutschen Gebiet gehörigen Ufer des Victoria-Njansa schon geschehen, aber aus mir unbekannten Gründen wieder aufgegeben. Es scheint mir sehr ratsam, dieVernichtung der Kroko dileier wieder aufzunehmen.

An solchen Stellen, wo die Glossinen regelmäßig Menschen antreffen und sich dann von diesen ernähren, z. B. an den Wasserentnahmestellen, die man oft in der Nähe der Dörfer am Seeufer findet, oder an Stellen, wo die Boote der Eingeborenen anzulegen pflegen, an viel benutzten Flußübergängen usw. können die Glossinen durch möglichst umfangreiche Abholzungen vertrieben werden.

Nach den hier auseinandergesetzten Prinzipien wird in Deutsch-Ostafrika gegen die Schlafkrankheit bereits energisch vorgegangen. Es kommen in dieser Beziehung bis jetzt drei Gebiete in Betracht, in welchen sich die Schlafkrankheit in größerem Umfange gezeigt hat: Kisiba, Shirati und Tanganjika. In Kisiba und Shirati sind bereits Lager für Schlafkranke errichtet und mit Ärzten besetzt, welche über die Schlafkrankheit und deren Bekämpfung unter meiner Leitung hinreichend informiert sind, und zwar befindet sich in Kisiba Stabsarzt $\mathrm{K} \mathrm{u} \mathrm{d} \mathrm{i} \mathrm{c} \mathrm{k} \mathrm{e} \mathrm{und} \mathrm{in} \mathrm{Shirati} \mathrm{Oberarzt} \mathrm{B} \mathrm{r} \mathrm{e} \mathrm{u} \mathrm{e} \mathrm{r.} \mathrm{Die}$ Bekämpfung der Schlafkrankheit am Tanganjika wird Stabsarzt F e $1 \mathrm{~d}$ m a n n übernehmen, welcher dorthin gehen wird, sobald er vom Kaiserlichen Gouvernement in Daressalam die erforderliche Ausrüstung erhalten hat. Jedem dieser Ärzte muß ein Sanitätsunteroffizier zur Hilfe beigegeben werden. Ich halte es auch für sehr zweckmäßig, diese Stationen, namentlich anfangs, wo es besonders viel zu tun gibt, mit zwei Ärzten zu besetzen, teils um in Erkrankungsfällen den Betrieb nicht ins Stocken kommen zu lassen, teils um auf diese Weise noch mehr Ärzte auszubilden und für den Fall, daß weitere Stationen errichtet werden müssen, geeignete Ärzte zur Verfügung zu haben. Ich halte es für sehr wahrscheinlich, daß am Tanganjika wegen der großen Ausdehnung des Seuchenherdes eine einzige Station nicht genügen wird. Es ist ferner zu befürchten, daß bei etwaigen weiterem Vordringen der Seuche in der Richtung nach Süden von. Shirati die große Insel Ukerewe ergriffen wird. Auf dieser Insel, welche gegen 30000 Einwohner haben soll, kommt in den Uferwäldern die Glossina palpalis vor, und es ist deswegen ein Einnisten der Seuche auf derselben sehr wohl möglich. Sie müßte von Shirati aus von Zeit zu Zeit besucht und auf Schlafkrankheit untersucht werden. Das kann aber ein einziger Arzt in Shirati, welcher mit der Beaufsichtigung der weit ausgedehnten Küste und der Buchten des Mori- und des Maraflusses vollauf zu tun hat, nicht ausführen, es sind dazu weitere Hilfskräfte erforderlich.

Nachdem so, wie ich annehme, alles in die Wege geleitet ist, was sich unter den gegebenen Verhältnissen zur Bekämpfung der Schlafkrankheit auf deutschem Gebiet tun läßt, halte ich die Aufgabe der Expedition für gelöst. Ich werde dieselbe daher zu Anfang Oktober nach Beendigung der noch im Gange befindlichen Arbeiten abschließen und am 14. Oktober von Mombassa die Heimreise antreten. 